\title{
The $\gamma$-glutamyltransferase activity and non-protein sulfhydryl compounds levels in rat kidney of different age groups
}

\author{
Przemysław Włodek ${ }^{1}$, Maria Sokołowska ${ }^{2}$, Olgierd Smoleński ${ }^{1}$ and Lidia Włodek ${ }^{2 \bowtie}$ \\ ${ }^{1}$ Department of Nephrology, Rydygier Hospital, Kraków, Poland; ${ }^{2}$ Institute of Medical \\ Biochemistry, Collegium Medicum, Jagiellonian University, Kraków, Poland
}

Received: 2 January, 2002; revised: 20 February, 2002; accepted: 22 April, 2002

Key words: kidney, aging, L-cysteine, glutathione, $\gamma$-glutamyl transferase, protein-bound cysteine, sulfane sulfur compounds, reactive oxygen species

\begin{abstract}
The present work was aimed to obtain information about age-dependent changes of $\gamma$-glutamyltransferase (GGT) activity and the levels of non-protein sulfhydryl compounds (NPSH) in rat kidneys. In addition, protein-bound cysteine (PB-Cys), sulfane sulfur compounds and reactive oxygen species (ROS) were estimated.

The results indicate that the activity of GGT and NPSH levels in the kidneys are reduced with age. At the same time, a significant increase in the level of protein-bound cysteine was observed. Simultaneously, the content of sulfane sulfur compounds was increased in the group of the oldest animals. These findings indicate that the capacity for extracellular glutathione degradation and, in consequence, the availability of cysteine for intracellular glutathione biosynthesis may be impaired. The increased PB-Cys level indicates potentiation of the thiolation reaction, i.e. development of protein-mixed disulfides. These results reveal age dependent disturbances in the thiol-disulfide equilibrium in the kidneys which leads to an imbalance between proand antioxidatory processes.
\end{abstract}

The intracellular glutathione (GSH) level depends upon the equilibrium between processes during which it is consumed and its biosynthesis limited by cysteine availability. The metabolism of GSH is closely connected to Meister's $\gamma$-glutamyl cycle (Meister, 1983) in which a pivotal role is played by membrane $\gamma$-glutamyltransferase (GGT), an enzyme whose active centre is directed extracellularly (Scheme 1). This enzyme participates in the salvage pathway of extracellular GSH by catalyzing its hydrolysis to amino-acid components, of which cysteine is used for intracellular GSH biosynthesis. Normally, due to

\footnotetext{
${ }^{\circledR}$ Corresponding author: Lidia Włodek, Institute of Medical Biochemistry, Collegium Medicum, Jagiellonian University, M. Kopernika 7, 31-034 Kraków, Poland, phone: (48 12) 422 7400, 618 8505, fax: (48 12) $4223272 .$.
}

Abbreviations: GGT, $\gamma$-glutamyltransferase; GSH, glutathione; PB-Cys, protein-bound cysteine; NPSH, non-protein sulfhydryl compounds; ROS, reactive oxygen species. 
cysteine toxicity, the physiological level of this amino acid in cells is very low (Puka-Sundvall et al., 1995), and in the plasma cysteine occurs mainly in the form of a disulfide - cystine. Consequently, the importance of the $\gamma$-glutamyl cycle lies in recovering and delivering cysteine (Scheme 1). The kidney is characterized by the highest GGT activity, nearly 900 tant cell antioxidant, depends upon GGT activity; hence this enzyme is considered to play an important role in the antioxidative defence systems of the cell (Kugelman et al., 1994). Lowered level of GSH impairs the above-mentioned functions to which it is indispensable; as a consequence, a deepening dysfunction of particular organs occurs.

Scheme 1

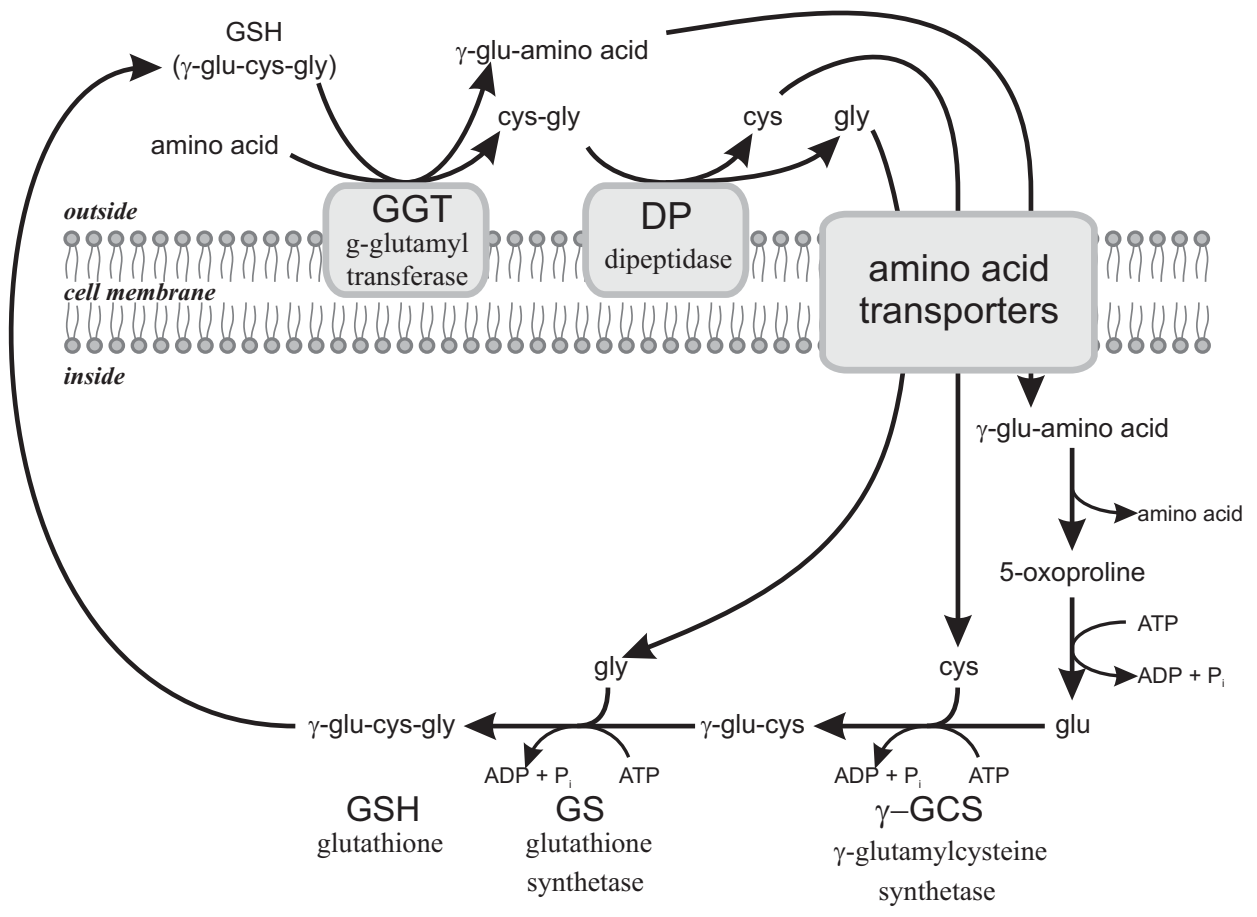

Scheme 1. $\gamma$-Glutamyl cycle (Meister, 1983)

times higher than in the liver (Hinchman \& Ballatori, 1990). This mode of recovering cysteine from GSH is particularly important for the kidney which, in contrast to the liver, can use methionine as a cysteine precursor to a small extent only (Sturman et al., 1970). Furthermore, very rapid turnover of cellular GSH has been demonstrated in the kidney and it is considered characteristic for this organ (Potter \& Tran, 1993). Thus the kidney plays a substantial role in the cleavage and interorgan transport of GSH, as well as in the maintenance of physiological levels of cysteine in the plasma (Meister, 1983). This means that the availability of cysteine, necessary for the biosynthesis of cellular GSH, the most impor-
Sulfane sulfur compounds contain a labile, highly reactive, reduce sulfur atom at a low oxidation state with a valence of 0 or -1 , covalently bound to another sulfur atom (Toohey, 1989; Iciek \& Włodek, 2001). These compounds include persulfides, polysulfides, thiosulfate and elemental sulfur. Sulfane sulfur compounds are formed in the anaerobic cysteine sulfur metabolism and participate in cell regulation processes through activation or inactivation of some enzymes. All those findings indicated a unique role of the kidney in cysteine and glutathione metabolism.

The present work was aimed to obtain information on how the GGT activity in the kidney changes with age of animals, and how these 
changes affect the non-protein sulfhydryl compounds (NPSH) (GSH and cysteine) level, as well as the concentration of cysteine bound in the form of protein-mixed disulfides and sulfane sulfur compounds - products of anaerobic cysteine metabolism.

We were prompted to undertake studies on rat kidney, since the highest cysteine concentration (Aebi \& Lauterberg, 1992) and the most intense GGT activity had been observed in that organ (Hinchman \& Ballatori, 1990; Potter \& Tran, 1993). Furthermore, an elevation in total plasma cysteine level as a consequence of renal failure had also been reported (Smolin et al., 1987; Włodek et al., 2001).

\section{MATERIALS AND METHODS}

Animals. The experiments were conducted on female Wistar rats obtained from the Department of Clinical Neurobiology, Free University in Berlin (Germany). The rats were sacrificed by cervical dislocation. The kidneys used in the experiments were from three age groups: young (3-month-old), middle-aged (19-month- old) and old (31-month-old). Each group consisted of 9 animals. The kidneys were quickly excised, washed with cold saline and subsequently frozen at $-70^{\circ} \mathrm{C}$ until further use. Immediately before the experiment, the kidneys were weighed, cut into pieces and homogenized in an ice-cold $0.1 \mathrm{M}$ phosphate buffer, pH 7.4.

Chemicals. L-Cysteine-HCl, 5,5' dithiobis(2-nitrobenzoic acid), reduced glutathione, dithiothreitol, NADPH, 2',7'-dichlorohydrofluorescin diacetate were provided by Sigma Chemical Co. (Deisenhofen, Germany). L-Glutamyl-4-nitroanilide was obtained from Boehringer Mannheim GmbH. Ninhydrin sodium and potassium cyanide were obtained from E. Merck (Darmstadt, Germany). Trichloroacetic acid was purchased from Ubichem plc Sigma Chemical Co. The remaining chemicals used were from Polish Chemical Reagents (P.O.Ch., Poland).
Methods. In rat kidney homogenates $(1 \mathrm{~g} / 4$ $\mathrm{ml}$ of $0.1 \mathrm{M}$ phosphate buffer, $\mathrm{pH} 7.4$ ), the level of reactive oxygen species (ROS) was determined according to the method of Bondy and Guo (1994); protein-bound cysteine was assessed according to Malloy et al. (1981), sulfane sulfur was determined by the method of Wood (1987), and the activity of glutamyltransferase was estimated by the method of Orlowski-Meister (1966). In the trichloroacetic acid supernatant of the kidney, determination of non-protein sulfhydryl compounds (NPSH; the sum of cellular GSH and cysteine) was carried out according to Sedlak \& Lindsay (1968).

Statistical analysis. The values shown in the figures are the mean \pm S.D. from nine separate experiments. Statistically significant differences between the three experimental groups were assessed by the ANOVA test.

\section{RESULTS AND DISCUSSION}

Both the renal GGT activity (young: $329 \pm$ $33.28 \mu$ moles of $p$-nitroaniline/g wet mass per min; middle: $284.8 \pm 15.15 \mu$ moles of $p$-nitroaniline/g wet mass per min; old: $252.65 \pm$ $20.65 \mu$ moles of $p$-nitroaniline, Fig. 1) and the NPSH level (young: $3.54 \pm 0.29 \mu$ moles/g wet mass; middle: $2.95 \pm 0.36 \mu$ moles $/ g$ wet mass; old: $2.64 \pm 0.23 \mu$ moles $/ g$ wet mass $)-$ which practically denotes the sum of reduced GSH and cysteine (Fig. 2) - fell in the successive age groups. At the same time, a significant increase in the level of covalently bound cysteine in the form of protein-mixed disulfides was observed (young: $1.19 \pm 0.096$ $\mu$ moles $/ g$ wet mass; middle: $1.47 \pm 0.36$ $\mu$ moles $/ g$ wet mass; old: $1.70 \pm 0.128$ $\mu$ moles/g wet mass) (Fig. 3).

On the other hand, the level of sulfane sulfur compounds, which reflects the intensity of anaerobic cysteine metabolism, was elevated in the group of the oldest animals as compared to the two younger ones (young: $0.68 \pm 0.037$ 


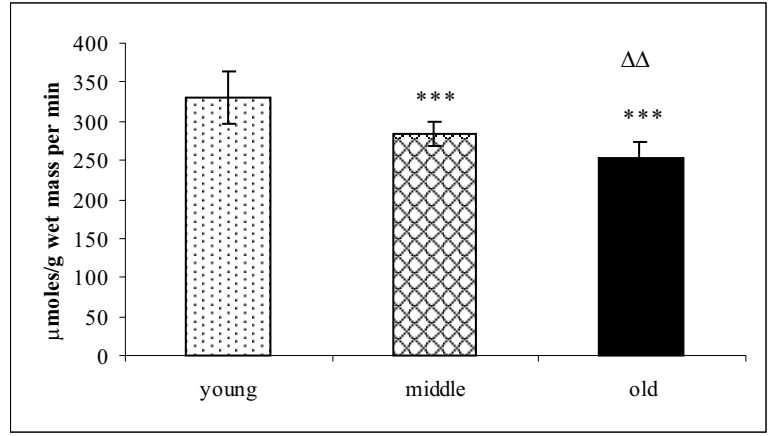

Figure 1. The activity of $\gamma$-glutamyltransferase in rat kidney of different age groups.

The enzyme activity was expressed in $\mu$ moles of $p$-nitroaniline/g of wet mass per min and represent means \pm S.D. of nine animals $\left({ }^{* *} P<0.001\right.$, significantly different from the first group (young); $\Delta \Delta P<$ 0.01 , significantly different from the second group (middle-aged)).

$\mu$ moles/g wet mass; middle: $0.62 \pm 0.06$ $\mu$ moles $/ g$ wet mass; old: $0.76 \pm 0.09 \mu$ moles $/ g$ wet mass, Fig. 4). Only in the group of the oldest rats (compared to the youngest), a slight

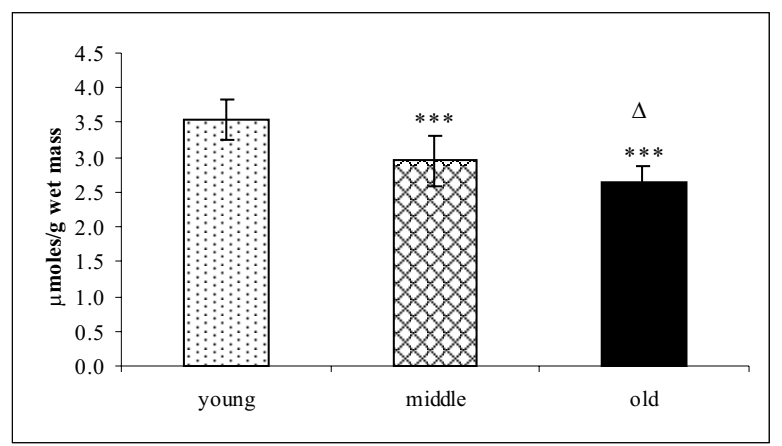

Figure 2. The level of non-protein sulfhydryl compounds (NPSH) in rat kidney of different age groups.

Values are expressed in $\mu$ moles of NPSH (GSH)/per g of wet mass and represent means \pm S.D. of nine animals ${ }^{* * *} P<0.001$, significantly different from the first group (young); $\Delta P<0.05$, significantly different from the second group (middle-aged).

increase in ROS levels was found (young: $0.100 \pm 0.007 \mu$ moles $/ g$ wet mass; middle: $0.109 \pm 0.011 \mu \mathrm{moles} / \mathrm{g}$ wet mass; old: $0.115 \pm$ $0.016 \mu$ moles $/ g$ wet mass, Fig. 5).

In parallel with aging, there can also be observed deepening dysfunctions of the kidneys,

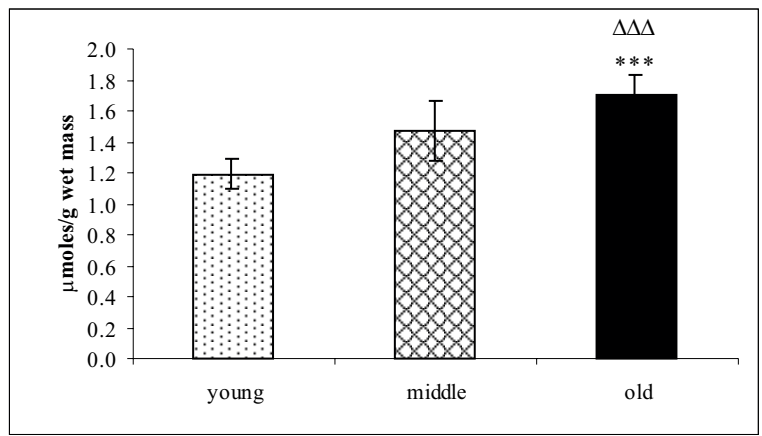

Figure 3. The level of protein bound cysteine in rat kidney of different age groups.

Values are expressed as $\mu$ moles of cysteine/g of wet mass and represent means \pm S.D. of nine animals $\left({ }^{* * *} P\right.$ $<0.001$, significantly different from the first group (young); $\Delta \Delta \Delta P<0.001$, significantly different from the second group (middle-aged).

which are thought to result chiefly from excessive peroxidation and glycosylation (Rodriguez-Puyol, 1998; Clark, 2000). These changes have been named tubulointerstitial nephropathy of the elderly.

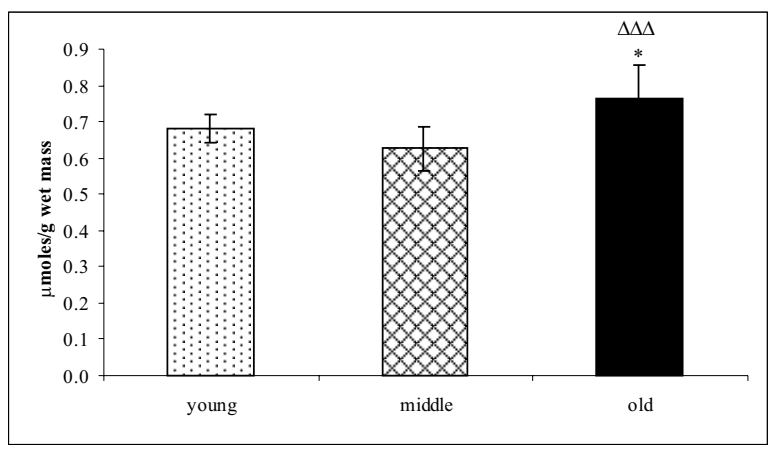

Figure 4. The level of sulfane sulfur compounds in rat kidney of different age groups.

Values are expressed in $\mu$ moles of potassium thiocyanate/g of wet mass and represent means \pm S.D. of nine animals $\left({ }^{*} P<0.05\right.$, significantly different from the first group (young); $\Delta \Delta \Delta P<0.001$, significantly different from the second group (middle-aged).

The presented results show that the activity of GGT and the NPSH level in the kidney decrease with aging of rats (Figs. 1 and 2) and fully confirm the results obtained by Jenkinson et al. (1991). Moreover, the authors noted two other facts: there was no age dependent 


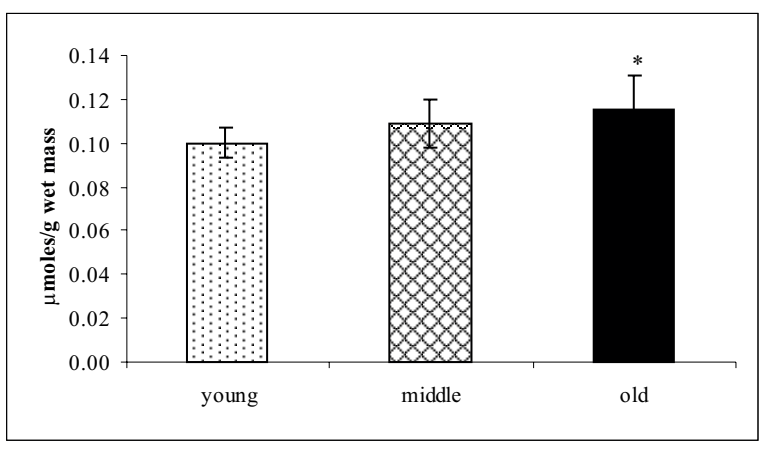

Figure 5. The level of reactive oxygen species (ROS) in rat kidney of different age groups.

Values are expressed in $\mu$ moles of dichlorofluorescein/ $\mathrm{g}$ of wet mass and represent means \pm S.D. of nine animals ( ${ }^{*} P<0.05$, significantly different from the first group (young)).

difference in the activity of glutathione peroxidase and glutathione $S$-transferase and, furthermore, plasma glutathione significantly increased in old animals as compared to the young. This finding indicates a disturbance of extracellular GSH degradation resulting in an impaired availability of cysteine for intracellular GSH synthesis. The age-dependent lowering of GGT activity in the kidney should thus lead to a diminished GSH level and, in consequence, to a decreased antioxidative defence of this organ. A further consequence of the diminution of the GSH level and GGT activity may be an impairment of the interorgan GSH transport and of the ability to maintain the physiological plasma cysteine level (Anderson et al., 1980). This suggests an insufficient supply of cysteine to all those tissues that cannot obtain cysteine via methionine metabolism.

The enzymatic profile of the kidney resembles that of the liver; only due to a lower mass of the former organ, its participation in total metabolism is correspondingly smaller (Lam et al, 1997). In spite of the overall resemblance of the enzymatic profiles of the kidney and the liver, the former shows an extremely high activity of GGT (Hinchman \& Ballatori, 1990) and a considerably lower activity of the enzymes converting methionine into cysteine
(Sturman et al., 1970). Thus the substantial enzymatic differences between these two organs are related to the metabolism of GSH and methionine, i.e. two processes connected with the biosynthesis of cysteine. Furthermore, the activities of GSH reductase and GSH peroxidase also diminish with age in mouse kidneys (Hazelton \& Lang, 1985). Our study shows a simultaneous diminution of the activity of GGT, another key enzyme connected with glutathione.

As regards GGT in the liver, a study by Speisky (1992) showed that its activity in old animals is enhanced compared to that in middle-aged ones. This implies that in the liver cleavage of GSH to cysteine enhances with age which, as a consequence, may facilitate the resynthesis of this tripeptide.

In the kidney, the activity of enzymes connected with anaerobic cysteine metabolism, i.e. cystathionase, 3-mercaptopyruvate sulfurtransferase and rhodanese, resembles that reported in the liver (Toohey, 1989; Stipanuk et al., 1990). On the other hand, the highest renal concentration of their metabolic products, i.e. compounds containing sulfane sulfur, is surprising and could not as yet be adequately explained (Ogasawara et al., 1994).

Our study shows that the concentration of sulfane sulfur compounds in the kidney is higher in the group of the oldest rats compared to the youngest and middle-aged animals (Fig. 4). These findings indicate that the diminished GGT activity and the lowered NPSH level in old kidneys are accompanied with an increased content of the metabolically extremely active sulfane sulfur compounds. Compounds with reduced sulfane sulfur are known antioxidants (Everett, 1995) and cell regulators (Toohey, 1989; Iciek \& Włodek, 2001). ROS level rises insignificantly only in the group of the oldest animals (Fig. 5). Hence the raised level of sulfane sulfur compounds in the kidneys of the oldest rats may be the ultimate line of antioxidative defence of this organ.

The process of aging in the kidneys is also accompanied with an elevated level of pro- 
tein-bound-cysteine in the form of mixed disulfides (Fig. 3). This covalent modification of proteins indicates enhanced oxidative alterations leading to changes in structure and function (Collison et al., 1986).

Cells with a high GGT activity and an elevated GSH level become resistant to toxic effects of both ROS and electrophilic xenobiotics. There is a close relationship between the toxicity of xenobiotics and the functioning of the kidney (Mühlberg \& Platt, 1999; Elfarra $\&$ Anders, 1984). The latter organ is responsible for their elimination via glomerular filtration, as well as for the biodegradation of glutathione S-conjugates due to a high activity of GGT (Hinchman \& Ballatori, 1990) and cysteine conjugate $\beta$-lyase (Kharasch et al., 1997). For the above reasons, concurrently with a decline in the renal function with age, the pharmacokinetics of many drugs is altered in elderly patients.

However, apart from the important biological role of GSH and GGT, under certain conditions they may participate in the bioactivation of glutathione S-conjugates, which may be dangerous to the kidneys. Cis-platinum, an antineoplastic drug with nephrotoxic side-effects leading to extensive acute necrosis of proximal tubules, may serve as an example here (Choie et al., 1981).

\section{R E F E R E N C E S}

Aebi E, Lauterberg BH. (1992) Divergent effects of intravenous GSH and cysteine on renal and hepatic GSH. Am J Physiol.; 263: R348-52.

Anderson ME, Bridges RJ, Meister A. (1980) Direct evidence for inter-organ transport of glutathione and that the non-filtration renal mechanism for glutathione utilization involves $\gamma$-glutamyl transpeptidase. Biochem Biophys Res Commun.; 96: 848-53.

Bondy SC, Guo SX. (1994) Effect of ethanol treatment on indices of cumulative oxidative stress. Eur J Pharmacol.; 270: 349-55.
Choie DD, Longnecker DS., del Campo AA. (1981) Acute and chronic cisplatin nephropathy in rats. Lab Invest.; 44: 397-402.

Clark A. (2000) Biology of renal aging in humans. Adv Renal Ther.; 70: 11-21.

Collison MW, Beidler D, Grimm LM, Thomas JA. (1986) A comparison of protein S-thiolation (protein mixed-disulfide formation) in heart cells treated with $t$-butylhydroperoxide or diamide. Biochem Biophys Acta.; 885: 58-67.

Elfarra AA, Anders MW. (1984) Renal processing of glutathione conjugates: role in nephrotoxicity. Biochem Pharmacol.; 33: 3729-32.

Everett SA. (1995) Antioxidant drug design: a comparison of thiol and perthiol antiradical and prooxidant radiation mechanism. In Biothiols in health and disease: Packer L, Cadens E. eds, pp 49-64. New York.

Hazelton GA, Lang CA. (1985) Glutathione peroxidase and reductase activities in the aging mouse. Mech Ageing Dev.; 29: 71-81.

Hinchman CA, Ballatori N. (1990) Glutathione-degradating capacities of liver and kidney in different species. Biochem Pharmacol.; 40: 1131-5.

Iciek M, Włodek L. (2001) Biosynthesis and biological properties of compounds containing highly reactive, reduced sulfane sulfur. Pol $J$ Pharmacol.; 53: 215-25.

Jenkinson SG, Duncan CA, Bryan CL, Lawrence RA. (1991) Effects of age on rat glutathione metabolism. Amer J Med Sci.; 302: 347-52.

Kharasch ED, Thorning D, Garton K, Douglas C, Hankin MA, Kitty CE. (1997) Role of renal cysteine conjugate $\beta$-lyase in the mechanism of compound nephrotoxicity in rats. Anesthesiology.; 86: 160-71.

Kugelman A, Choy HA, Liu R, Shi MM, Gozal E, Forman HJ. (1994) Gamma-glutamyltranspeptidase is increased by oxidative stress in rat alveolar L2 epithelial cells. Am J Resp Cell Mol Biol.; 11: 586-92. 
Lam YWE, Banerji S, Hatfield C, Talbert RL. (1997) Principles of drug administration in renal insufficiency. Clin Pharmacokinet.; 32: $30-57$.

Malloy MM, Rassin DC, Gaull GE. (1981) A method for measurement of free and bound plasma cysteine. Anal Biochem.; 113: $407-15$.

Meister A. (1983) Metabolism and transport of glutathione and other $\gamma$-glutamyl compounds. In Functions of glutathione: biochemical, physiological, toxicological and clinical aspects. Larsson A, Orrenius S, Holmgren A, Mannervik B. eds, pp 1-22. Raven Press, New York.

Mühlberg W, Platt D. (1999) Age-dependent changes of the kidneys: pharmacological implications. Gerontology.; 45: 243-53.

Ogasawara Y, Isoda S, Tanabe S. (1994) Tissue and subcellular distribution of bound and acid-labile sulfur and the enzymatic capacity of sulfide production in the rat. Biol Pharm Bull.; 17: 1535-42.

Orlowski M, Meister A. (1966) Isolation of $\gamma$-glutamyl transpeptidase from hog kidney. $J$ Biol Chem.; 240: 338-40.

Potter DW, Tran T. (1993) Apparent rates of glutathione turnover in rat tissues. Toxicol Appl Pharmacol.; 120: 186-92.

Puka-Sundvall M, Eriksson P, Nilscon M, Sondberg M, Lehmann, A. (1995) Neurotoxicity of cysteine: interaction with glutamate. Brain Res.; 705: 65-70.
Rodriguez-Puyol D. (1998) The aging kidney. Kidney Intern.; 54: 2247-65.

Sedlak J, Lindsay RH. (1968) Estimation of total protein bound and nonprotein sulfhydryl groups in tissues with Ellman's reagent. Anal Biochem.; 25: 192-205.

Smolin LA, Laidlaw SA, Kopple JD. (1987) Altered plasma free and protein bound sulphur amino acid levels in patients undergoing maintenance hemodialysis. Am J Clin Nutr;; 45: $737-45$.

Speisky H. (1992) Age-dependent removal of circulating glutathione by rat liver: role of gamma-glutamyl transferase. Age.; 15: 104-7.

Stipanuk MH, De La Rosa J, Hirschberger L. (1990) Catabolism of cysteine by rat renal cortical tubules. $J$ Nutr.; 120: 450-8.

Sturman JA, Rassin DK, Gaull GE (1970) Distribution of transsulphuration enzymes in various organs and species. Int $J$ Biochem.; 1: $251-3$.

Toohey JJ. (1989) Sulphane sulphur in biological systems: a possible regulatory role. Biochem J.; 264: 625-32.

Włodek P, Iciek MB, Miłkowski A, Smoleński OB. (2001) Various forms of plasma cysteine and its metabolites in patients undergoing hemodialysis. Clin Chim Acta.; 304: 9-18.

Wood L. (1987) Sulfane sulfur. Methods Enzymol.; 143: 25-9. 